\title{
Keeping our heads about our heads
}

Controversial questions are currently being raised about psychosurgery.

Keith Oatley of the Laboratory of Experimental Psychology at the University of Sussex comments on some of the issues

IN THE last few weeks public notice has been drawn to the use of physical and surgical interventions in the treatment of "mental illness". The release of the film "One Flew Over the Cuckoo's Nest", in which both electro-convulsive treatment and lobotomy were portrayed as techniques of punishment and control, was followed by a television documentary of a woman who had bouts of violent behaviour, and who was operated on by a neurosurgeon to remove part of her hypothalamus. The results were then reported of an Inquiry which upheld allegations made at a "typical" British mental hospital that electroconvulsive treatment was given "casually" and on "a disturbingly large number" of occasions. All this comes at a time when the Royal College of Psychiatrists is planning a controlled study of psychosurgery on 200 patients in the London area.

Under these circumstances it seems appropriate to consider some important issues raised by psychosurgery: the attempt to control entirely mental and behavioural symptoms (with no organic pathology) by physical stimulation or lesioning of the brain. Two fairly extreme positions can be taken up, both with a good measure of enthusiasm, righteous indignation, and often some reluctance to examine the evidence.

One position is that psychosurgery is harbaric, infringes patients' rights, and produces irreversible damage to the brain (how can less brain be better than more?). What justification is there in any case, it is asked, for supposing that people who have difficulties in living are "ill" or need to have some part of their brain stimulated or removed?

At the other extreme some members of the medical profession claim that psychiatric disturbances are illnesses with physical causes, that it has been shown that for some of these, intervention in the brain produces alleviation of symptoms, and even cures, and that doctors are in a position both to recommend, carry out and evaluate such treatment. (This latter point for instance was the conclusion of The Times medical correspondent Dr Tony Smith. "Only another doctor can be certain that a line of medical treatment was inadvisable, unethical, or simply wrong".)
Neither position squares well either with our physiological, psychological or sociological understandings of psychologically disturbed, or disturbing behaviour. Three kinds of consideration seem to be most relevant. First, there are the reported results of psychosurgical procedures which have already been undertaken. Secondly, there is evidence from experimental animal work (from some of which the psychosurgical techniques were originally derived), and from experimental brain damage in people. Finally, there are some fundamental considerations of how people, including doctors and patients, ought to behave towards one another, backed if necessary with legal sanctions. It is worth considering one illustrative example from each of these.

In a recent issue of the British Journal of Psychiatry (128, 226-240; 1976), N. Mitchell-Heggs, D. Kelly and A. Richardson of St George's Hospital Medical School report on 66 patients given psychosurgery, and followed up over a mean period of 16 months. The operations were multifocal. Lesionmaking electrodes were placed sterotactically in 10 sites of each brain (typically three in each frontal lobe, and two in each cingulum). The thermocoagulative lesions were aimed at various limbic tracts, and each estimated at $6 \mathrm{~mm}$ in diameter. Before and after operation patients were assessed on an impressive number of scales and inventories, and in 28 cases by a semi-independent assessor (a retired psychiatrist). The report was that $73 \%$ of the patients were improved at 6 weeks and $76 \%$ at 16 months. The paper is excellently detailed by the usual standards of such reports. The authors take pains to discuss the prerequisites for the operation (that is, an exhaustive trial of less statistics about their assessments.

Various comments can however be made. There is no control group, for example, and with the best will in the world the assessment is done by people who have a strong interest in reporting success of the procedure. Also, although one gets the impression that many patients did benefit substantially from the treatment (various remarks being made about "returning to work" and so on), the measures of improvement did not include assessment of analysis of, for example, accidental radical treatments), and give a lot of whether the patients were improved with respect to their own objectives in life, or with respect to whether they were easier to contain in institutional care. Moreover, many of the scales used were heavily biased. Clinical rating scales, for instance, had three categories for "improved", one for "unchanged" and one for "worse". It is known that to approximate to fairness, rating scales should be symmetrical about the null hypothesis of "no change".

Regarding the experimental evidence (mostly from animals), this indicates that brain anatomy is no less variable than the anatomy of human faces, that sterotaxic placement of electrodes is in any case subject to errors (for example, of electrodes bending or being misdirected and thus ending up in places other than those planned). Even disregarding these there is no evidence that parts of the brain are related in a one-to-one fashion or any other simple way to categories of behaviour such as aggression or obsessionality. (The best review of these matters in the context of psychosurgery is by E. Valenstein, Brain Control (Wiley; 1973)).

Finally, although careful consideration of the experimental evidence from animal work would dissuade one from attempting psychosurgery, it does already take place, and some doctors believe it benefits some extremely disabled people considerably. If psychosurgery is to take place at all, what we do need is a proper trial, with proper controls, with informed consent of all subjects, and the participation of people (other than medics.) who know how to design and assess such experiments.

The trial should compare a group of patients who have been referred for psychosurgery (on the basis of severe disablement and failure of previous treatments). Half should have the psychosurgery, and the other half a further attempt at the best available non-surgical treatment (perhaps a combination of chemotherapy and behaviour therapy). All participants should be given supportive psychotherapy and rehabilitation help throughout. Assessment before and after treatment should be undertaken by several independent people, of different psychiatric persuasions, and without knowledge of what treatment each patient is given.

There are ethical problems; but these are less questionable than the current practice of doctors carrying out research without informed consent of all subjects in ill controlled studies which continue to leave room for doubt about the efficacy of procedures which then continue to be practised. 